\section{Better informed: an overview of health informatics}

\author{
P. A. Reynolds, ${ }^{1}$ J. Harper, ${ }^{2}$ A. M Jenner ${ }^{3}$ and S. Dunne ${ }^{4}$
}

IN BRIEF

- Gives definitions of healthcare informatics $(\mathrm{HCl})$ and explains the significance and growth of this aspect of healthcare.

- Traces the historical development of $\mathrm{HCl}$ and suggests that the two major aspects of its usage are for health management and audit, and provision of clinical information and diagnostics.

- Describes recent developments in $\mathrm{HCl}$, together with the plans for $\mathrm{HCl}$ in NHS dentistry.

Healthcare informatics is increasing in importance both for healthcare administrators and medical and dental practitioners. Governments across the developed world are initiating major national health IT programmes. At the same time, future best medical and dental practice will increasingly depend on computer-based support tools, although disagreement remains about the effectiveness of current support tools. Over the longer term, future informatics tools, combined with other medical and dental technology modalities, promise more adaptive, patient-focused and efficient healthcare and education for the practitioner and the patient.

\section{E-LEARNING IN DENTISTRY}

Section A: Teaching and technology

1. A description of the new technologies used in transforming dental education

2. Seeing is believing: dental education benefits from developments in videoconferencing

3. Webcasting: casting the web more widely

4. Top of the pops - CD-ROM and DVDs in dental education

Section B: Informatics: better informed by systems and services

5. Better informed: an overview of health informatics

6. Better informed in clinical practice a brief overview of dental informatics

7. Digital clinical records and practice administration in primary dental care

Section C: Impact of e-learning in

dental education

8. Remember the days in the old school yard: from lectures to online learning

9. An intricate web - designing and authoring a web-based course

10. The many faces of interaction

11. Supporting the learner and teacher online

12. Making a mark - taking assessment to technology

13. Continuing professional development and ICT: target practice

14. Assuring quality

Section D: A connected future

15. Nine years of DentEd: a global perspective

16. A vision of dental education in the third millennium

\section{INTRODUCTION}

In an earlier article $^{1}$ in this series, its authors explained that informatics, although not in itself a technology is a key element in e-learning. Some may question the place of informatics in elearning, but if the quotation they used to illustrate their claim is correct, ie "elearning is defined in a way that uses information and communication technologies (ICT),, 2 then it is appropriate that this series should address the topic, especially as dental informatics has been seen as 'the application of computer and information sciences to improve dental practice, research, education (our italics) and management.'

\footnotetext{
1*Professor of Dental Education, Centre of Flexible Learning in Dentistry, King's College London Dental Institute, Floor 3, Strand Bridge House, 138-142 The Strand, London, WC2 $1 \mathrm{HH}_{;}{ }^{2} \mathrm{IT}$ Manager, The Dental Institute at Guy's, King's and St Thomas' NHS Hospitals, King's College Hospital, Denmark Hill, London, SE5 9RW; ${ }^{3}$ Visiting Professor, The University of Liverpool School of Dentistry, Pembroke Place, Liverpool, L3 5PS; ${ }^{4}$ Professor of Primary Dental Care, The Dental Institute at Guy's, King's and St Thomas' NHS Hospitals, King's College Hospital, Denmark Hill, London, SE5 9RW ${ }^{*}$ Correspondence to: Professor P. A. Reynolds Email: p.a.reynolds@kcl.ac.uk
}

\section{Refereed Paper}

DOI: $10.1038 /$ bdj.2008.150

${ }^{\circledR}$ British Dental Journal 2008; 204: 259-264
It is only recently that, after years of relative neglect by governments, health informatics has become the subject of some of the world's largest public sector procurement programmes. In many countries, governments are initiating large-scale national electronic health record programmes involving many billions of pounds expenditure. In the British Isles, these health record programmes will be extended over time to include dental practices.

But despite the vast investment now focused on national health record programmes, healthcare informatics is not limited to electronic record systems. Other areas, such as clinical decision support systems, have attracted major interest from both researchers and practitioners - although the efficacy of clinical decision support systems remains a subject of debate.

Over the next decade, new information technologies and new clinical practices will have a marked impact on the shape of healthcare informatics. Of the former, workflow management and text mining are likely to have a significant impact. Of the latter, the arrival of desktop genetic and protein testing in clinical practice will open up the opportunity 
for 'individualised medicine', which will depend on new developments in adaptive workflow and flexible networks of care and education.

\section{DEFINITION}

The term 'informatics' attracts a number of differing interpretations. Some are scientific: 'Informatics studies the representation, processing, and communications of information in natural and engineered systems. The central notion is the transformation of information whether by computation or communication, whether by organisms or artefacts.'

Other definitions ${ }^{2}$ reveal its links to information and communications technology (ICT): it is 'The sciences concerned with the gathering and manipulating and storing and retrieving and classifying recorded information. ${ }^{4}$ It 'develops new uses for information technology' and 'is also interested in how people transform technology, and how technology transforms us. ${ }^{5}$

In healthcare, its derivative, health informatics, is seen as 'The knowledge, skills and tools which enable information to be collected, managed, used and shared to support the delivery of healthcare and to promote health. ${ }^{6}$ Whatever the definition, the chances are that its emanation is often prosaic and an accepted part of everyday routine: in healthcare, think patient records.

\section{HISTORY}

The forerunners, and the main impetus, to the development of health informatics were the healthcare audit systems introduced by Florence Nightingale, and the medical records system devised by the Mayo Clinic. Nightingale (Fig. 1) believed passionately in the role of statistics in guiding health policy and practice. She became a pioneer in epidemiological methods, and devised statistical diagrams that made complex data clear to the most innumerate policy maker. Her approach underlies integrated care pathways, one of the main driving forces behind major informatics programmes such as England's National Programme for IT. ${ }^{7}$

The Mayo Clinic in Rochester, New York, USA, initiated an equally revolutionary development in 1907 with the

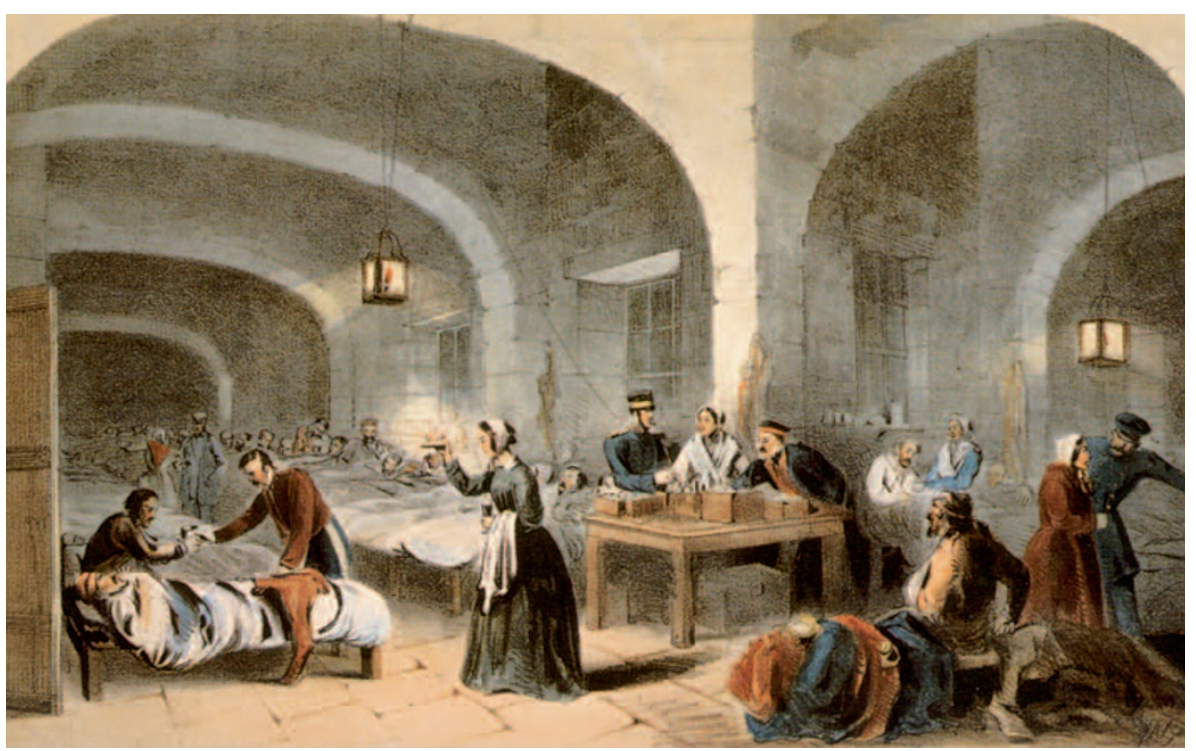

Fig. 1 Florence Nightingale in the Military Hospital at Scutari. Lithograph by J. A. Benwell, 1855. Courtesy of the Florence Nightingale Trust, London

introduction of a dossier medical records system to the hospital. All a patient's data, from both clinic visits and hospital stays, was entered into a single file which was stored in a central repository. This file with the patient's complete history was accordingly available every time the patient visited the hospital. Just as importantly, a diagnostic index was also devised that allowed doctors and researchers to locate all records relating to a particular disease or condition. In fact this was the first clinical disease database, over forty years before the commercial introduction of the computer. ${ }^{8}$

The system's indices were adapted in the 1930 s as part of a coding index known as the Berkson Codes, after its developer. This system made it possible to classify more than 20,000 diseases and sites in the body.

Nightingale's healthcare audit systems, and the later clinical records system, point to the two main directions taken by health informatics since: one to health management and audit; the other to clinical information and diagnostics. That divergence, and tension between the two goals, remains a key aspect of health informatics.

\section{Into the computer age}

The first use of computers in healthcare was by a dentist, Dr Robert Ledley. In 1950, he used a computer at America's National Bureau of Standards, one of only a handful in the world, to number-crunch data for dental research projects.

The concept of the electronic medical record arrived soon after at the National Bureau. The two main pioneers in its development were Ledley and his colleague Lee Lusted. ${ }^{9}$ The latter's paper, 'Medical progress - medical electronics' in the New England Journal of Medicine, 1955, was one of the first statements of the need for the computerisation of health records. ${ }^{10}$

One of the very first medical records systems was introduced in 1960 at the Oregon Regional Primate Research Center, where it was used for veterinary research. Human medical records systems followed in the later 1960s, most notably at LDS Hospital in Utah and the Kaiser Permanente health system in California. The LDS system, developed on an IBM mainframe for the hospital's intensive care unit, can claim to be the first critical care clinical information system. Later work for the Kaiser Permanente organisation was one of the first triage systems developed for casualty and out-patient use. ${ }^{9}$

However, most health informatics research in the 1950s and 1960s focused on the promising area of artificial intelligence. Some of the earliest expert systems were designed for medical use, such as MYCIN, developed in the mid-1950s, and INTERNEST-I.

Although expert systems appeared to hold out great hope for rendering vast 
amounts of expert knowledge accessible to practitioners, in use the early systems proved disappointing. The logical schema they applied proved too simplistic for complex medical cases. More complex analytical models have been circumscribed by the limitations of hardware and storage.

\section{Towards the electronic patient record (EPR)}

The most notable current development in health informatics is the creation of national e-health infrastructures in many of the main developed countries. At present, the single largest e-health programme is that of the NHS in England. However, this could be dwarfed in time by both the German and the US programmes, which cover larger populations. The French programme, which is linked to the automation of the entire social welfare benefits and pensions system, is also potentially larger than England's, involving the creation of a national electronic infrastructure for integrated benefits assessment and healthcare practitioner payment by activity across the entire health and social security infrastructure.

All European Union (EU) countries are committed to an e-Health Action Plan, first defined at the Lisbon InterGovernmental Conference. This commits governments to creating electronic infrastructures for health and ensuring portability of health data and national insurance information across all EU health administrations.

The English programme will provide regional electronic health records (EHR), known as the national care record service. The country has been divided into five regions and each region will be connected into a national data spine. The result is that GPs, hospitals and clinics across England should have access to patient records wherever the patient resides.

\section{ELECTRONIC HEALTH RECORDS (EHR) AND INTEGRATED CARE PATHWAYS}

A principal impetus towards the adoption of comprehensive electronic health records is the goal of achieving integrated care pathways (ICPs). ICPs provide a framework and a timeline for the delivery of specific, protocol-based care to patients with particular conditions or symptoms.

Applying informatics to ICPs powerfully enhances the scope and the efficacy of the ICP approach. Computer-based ICPs are able to incorporate a wider and more complex set of disease protocols, and apply a more patient-focused and adaptive approach to the creation of individualised patient care pathways.

Within the context of national health record programmes, computer-based ICPs provide a tool to incorporate local and national guidelines into clinical practice, enable complex pathways across networks of care providers, and provide the means for managing clinical risk, benchmarking and clinical audit. ${ }^{11}$

\section{EHR and dentistry}

Many EU countries are including dental services and organisations in their plans for a national electronic health record infrastructure. This largely depends on whether or not there is a major difference in funding formula between dentistry and medical care. So both in countries where dentistry is fully centrally funded and in those where the entire state healthcare system is subject to a decentralised funding mechanism, dentistry has been included as an integral EHR service.

Within the UK, Scotland, where the Scottish Executive from the outset has seen the future of dentistry as wholly within the Scottish NHS, dental services have been included in pilot schemes to develop an e-health infrastructure. One such pilot is the Scottish TeleOrthodontic Project (STOP), which provides an EPR service for hospital-based orthodontist consultants and local dental practices across the Highlands and Islands and Dumfries and Galloway regions. The STOP project also provides for an EHR mechanism for structured data interchange between EPR systems. This approach is consistent with Scotland's strategy of building a national EHR infrastructure up from local EPR implementations.

As part of the EU's e-Health Action Plan 2002, France, Germany and Italy will be developing a common standards-based smartcard electronic patient record as a joint EU pilot project. These smartcards will represent a next generation development of each country's existing card systems, with considerably more memory and functions. Data held will include demographic, identifier, and a subset of an EPR. When these new smartcards come into operation there will be a common dental record between all three countries.

England has the most ambitious national EHR programme of any European country. At present, dental services are not part of its core dataset requirements as set out by the National Programme for IT Office.

In September 2000, the Department of Health published Modernising NHS dentistry - implementing the NHS plan. This set out the objective of a more patientfocused dental service, in line with the NHS plan.

In August 2001, the IT task group of the NHS Dentistry Modernisation Steering Group called for the development of a detailed national IT strategy for dentistry in England. This would include a dental EPR, integration into the national EHR infrastructure, development of clinical decision support services for NHS dentistry, and the introduction of standardised clinical indicators to support dental clinical governance.

In 2002, the NHS published NHS dentistry: options for change, ${ }^{12}$ which called for the closer integration of dental services into local NHS healthcare infrastructures and the development of managed clinical dental networks by primary care trusts (PCTs). The document argued that linking dental EPR into regional EHR and PCT planning systems was 'fundamental' to the delivery of the Options for Change programme.

In October 2002, the Department of Health published An information technology strategy for NHS dentistry in the 21 st Century. ${ }^{13}$ Identifying the reintegration of dental services into the NHS as the over-riding theme of Options for Change, the document called for dental EPRs to be developed within an NHSwide EPR and EHR infrastructure. The report's authors conclude, however, that the dental EPR should be developed independently of the mainstream 
medical EPR, since the requirements for a dental service record are significantly different from those of medical practice. The report also underlined the importance of developing dental service integrated care pathways (ICPs) to extend evidence-based practice and clinical governance in dentistry.

$£ 30$ million was ear-marked for information technology to integrate dentistry within the national IT programme. A draft specification of requirements was created in 2005 but the project has been delayed as NHS Connecting for Health concentrates on the larger medical implementation of the national programme.

\section{CLINICAL DECISION SUPPORT}

For doctors, one of the most interesting classes of healthcare informatics is clinical decision support systems (CDSS). These systems aim to provide functions that directly benefit the doctor's main role, supporting the diagnosis and management of patients. By contrast, the massive investment in EHR and EPR technologies going in to the spate of e-health initiatives, such as England's National Programme for IT, has been criticised by many doctors' leaders as being of only marginal benefit for clinicians.

The degree to which CDSS have benefited doctors and their patients is, however, debatable. While some researchers have pointed out significant gains in both diagnostic accuracy and patient safety from CDSS, a significant number of other researchers and, recently, the programme's office, have claimed the evidence does not support such claims.

Clinical decision support systems have found a wide range of applications since their introduction in the late 1970s. These include diagnostic support, laboratory support, automated patient monitoring, prescribing support, machine learning and research support. In addition, clinical decision support systems have been found to help medical education, communications between doctors and patients and family, and compliance with integrated care pathways.

Most of the earliest efforts saw diagnostic support as the principal objective of clinical decision support systems. The early systems were hampered by a number of design flaws, including poor interface design, limited knowledge bases, over simplistic rules-based reasoning that could not comprehend complex medical conditions, and poor integration with clinical working practices. The systems that have proved most successful have been tightly focused on specific disease areas, have had access to a comprehensive domain knowledge base, and have effective integration into other clinical workplace tools such as HISS (hospital information and support systems), patient management systems, or clinical information systems. More recently, neural and probability network reasoning systems have provided diagnostic support better suited to evaluating complex multi-disease and multi-organ failure state conditions.

Despite diagnostic support systems being the focus of research efforts, CDSS has had perhaps most effect in two other applications: prescribing support and laboratory automation.

Laboratory-based CDSS are routinely used to generate pathology reports in hospitals. Systems that have been validated in the research literature include the PUFF system, introduced in $1977 .{ }^{14}$ PUFF is used for pulmonary function tests. Another is the Pathology Expert Interpretative Reporting System (PEIRS), which is used for interpreting a range of endocrine and haematocritic tests. ${ }^{15}$

In clinical practice, CDSS' strongest claim for effectiveness has been in the area of prescribing support. Studies have shown that prescribing CDSS can significantly improve accuracy ${ }^{16}$ and reduce adverse drug events. ${ }^{17}$ However, these findings have been challenged by other researchers. ${ }^{18,19}$

In December 2004, the National Programme for IT Office asked NICE, the National Institute for Health and Clinical Excellence (http://www.nice.org.uk), to carry out a review of all CDSS in use in the NHS. It claimed that many systems currently in use may be unreliable and could even endanger patients' lives by failing to warn adequately about drug interactions. NICE's review will include even very recent systems such as Isabel.*

*Isabel Healthcare Ltd, PO Box 244, Haslemere, Surrey, GU27 1WU, UK
Over the years, researchers have cast doubt on the basis for CDSS' claims, especially in the area of diagnostic support. A number of literature reviews, the most recent by UNSW's Vitali Sintchenko in 2003 for the Australian Government, ${ }^{20}$ have found that many studies reporting benefits of diagnostic CDSS were poorly structured and relied on dubious reporting techniques.

In 2001, a meeting of the American Medical Informatics Association, the AMIA, concluded that CDSS had had only a modest effect on improving prescribing accuracy, while their effect on clinical outcomes was indeterminable, but probably minor. The meeting decided that a new category of CDSS software was required to increase the effectiveness of CDSS and meet the challenges of evidence-based medicine. This new class of software has been labelled 'evidenceadaptive CDSS' ${ }^{21}$

The AMIA has set out two key technology challenges for evidence-adaptive CDSS research: automatic capture of both literature and practice-based research into standardised machineinterpretable formats, and the development of methods and technology for applying research to individual patients at point of care.

With literature-based evidence, the most obvious first step is to incorporate the efficacy studies produced by evidence-based medicine (EBM) researchers. However, problems surround much of this EBM literature. Its scope covers only a fraction of the available literature, and many have been beset by methodological and reporting problems. Moreover, any human effort is fighting a losing battle with an exponentially growing clinical research literature.

Evidence-adaptive CDSS researchers believe that CDSS will only support EBM when they can 'keep up' automatically with the literature. This means that evidence-adaptive CDSS must be able to monitor the entire body of medical literature, identify studies of high quality and incorporate those findings into automated patient assessments and recommendations.

Much of this will be dependent on research in other fields, especially the developing area of text mining, or 


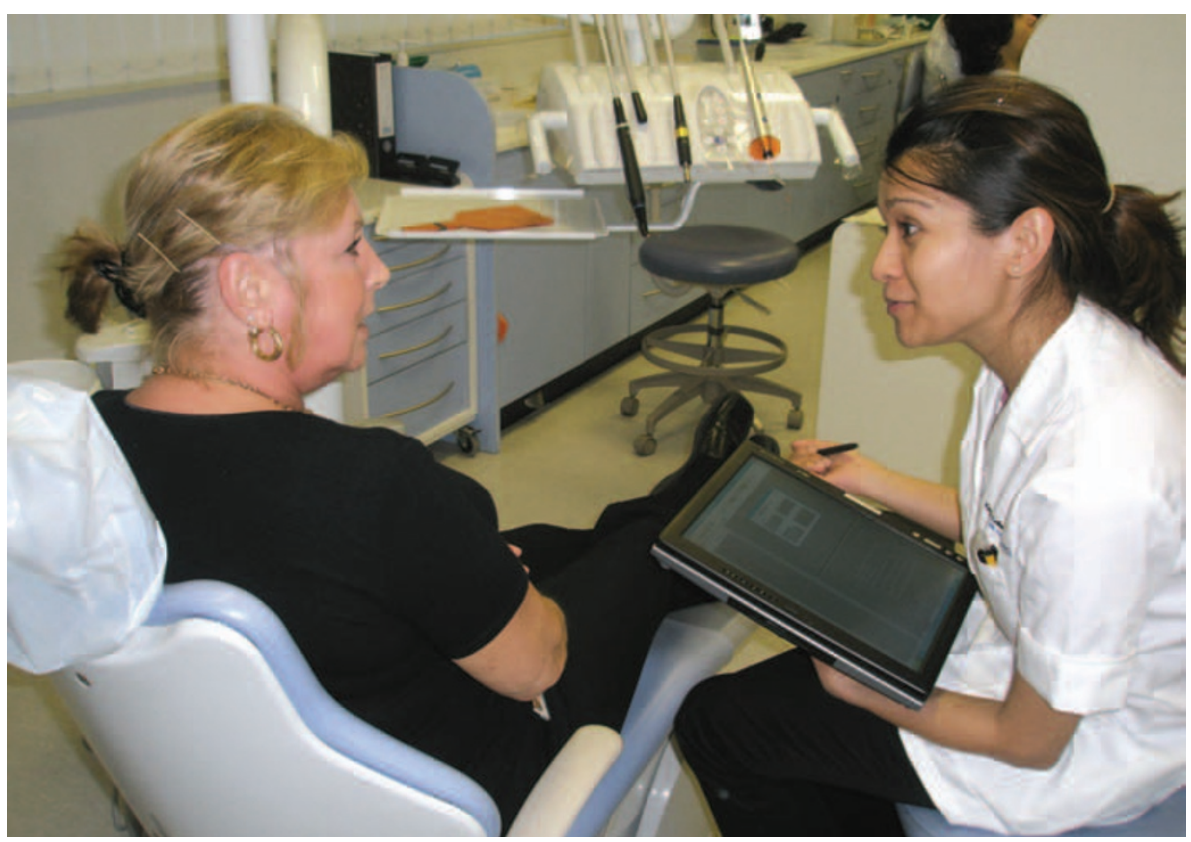

Fig. 2 Future chairside computing may include portable touch screen technology such as the tablet

textomics. Future research on evidenceadaptive CDSS will almost certainly look to incorporate the growing global evidence base from genomic and proteomic databases and annotation systems.

\section{THE FUTURE}

The main effect of the introduction of the NHS Connecting for Health national e-health infrastructure on practitioners over the next ten years is likely to focus firstly on increasing patient choice and convenience through 'choose and book' and the electronic prescription service (EPS), picture archiving and communication system (PACS) and improving practice experience through chairside computing and access to patient records (Fig. 2). Following this will be the close integration of integrated care pathways (ICPs) into clinical practice. The starting point for this should be a fully integrated oral health assessment with electronic decision tree built into existing clinical systems.

The way forward for informatics in dentistry should be the development of a specification for primary dental care systems that not only reflects NHS information standards but also the needs of a modern health service by supporting ICPs and oral health assessments. By providing such a specification, system suppliers will then be able to build systems that both support the delivery of care for patients and are compliant with national information systems. The business case for the development of this specification is currently under discussion but no substantial development work has yet taken place.

Beyond the infrastructure, several other health informatics developments are likely to affect clinical working practices. The most immediate of these is the arrival of clinical workflow management. These systems are the result of the integration of biodata and image management applications onto standard commercial computing environments. Clinical image management and patient data monitoring systems typically reside on special stand-alone systems that allow special calls to be executed directly to the system microcode. The recent development by some medical equipment vendors of an imaging layer that integrates directly into commercial operating systems, allowing high performance microcode access without destabilising other applications, opens up the possibility of extending the functionality of specialist clinical imaging and monitoring systems right across a commercially standard hospital network.

Several large vendors are also developing 'individualised' or 'personalised medicine' information systems. The objective is to integrate the results of genomic evaluation systems into CDSS systems to direct testing and patient management. Medical testing will be guided to the conditions suggested by a patient's DNA tests. To succeed, individualised medicine must integrate CDSS diagnostic support with interpretation of genomic and proteomic data.

One technology that has emerged out of genomic research is textomics: efficient text analysis applications that were developed to interpret the very long data files generated by efforts to map the human genome. Textomics and text mining could revolutionise clinical practice by taming burgeoning medical literature and discovering undetected causes and cures for many diseases. Text mining is integral to the development of evidence-adaptive CDSS.

The University of Chicago has developed text mining techniques to make several medical breakthroughs. ${ }^{22}$ Swanson's first discovery related to Reynaud's syndrome: a suggestion that fish oils could help improve the condition - which was subsequently confirmed by independent research. ${ }^{23}$ Swanson also discovered that magnesium often prevented the onset of spreading depression. From this, he suggested that lack of magnesium could be a factor in causing migraines, a suggestion also subsequently confirmed. ${ }^{24}$

The iMed Health Informatics School at Washington University has developed LittLinker. Based on Swanson's Open Discovery methodology, LittLinker is designed to discover new causal links between biomedical terms. One project that has come out of their work is a cancer outpatient support system that simplifies many of the choices and tasks facing cancer sufferers under outpatient care.

The University of Texas is text mining the clinical literature to find new applications for existing drugs. Since new drugs take about 15 years and about $\$ 1$ billion to reach the market, text mining could be a much faster and cheaper way to treat conditions with drugs that already have FDA approval. Using text mining, the team has already identified chlorpromazine, a treatment for psychotic disorders, as a new treatment for cardiac hypertrophy. This is all part of creating a wider knowledge base enabling a better informed practitioner. The 
eventual aim of text mining research is to integrate their tools into desktop systems used by practising clinicians.

\section{SUMMARY}

Having long been relegated to the healthcare sidelines, today healthcare informatics is becoming an essential platform for healthcare delivery which involves clinicians, students and patients. Key healthcare objectives, such as integrated care pathways, evidence-based medicine and protocol-driven clinical practice, are now increasingly seen to depend on the widespread adoption of healthcare informatics throughout both primary and secondary healthcare.

In the future, new technologies such as text mining, individualised medicine and clinical workflow point the way to more flexible, adaptive and patient-centred ways of delivering advanced healthcare.

1. Feeney L, Reynolds PA, Eaton K A, Harper J. A description of the new technologies transforming dental education. Br Dent $J$ 2008; 204: 19-28.
2. Laurillard D. Moving towards a unified e-learning strategy. London: Department for Education and Skills, 2004. www.dfes.gov.uk/consultations/ downloadableDocs/towards\%20a\%20unified $\% 20$ e-learning \%20strategy.pdf

3. Website of the Faculty of Health Informatics, Royal College of Surgeons of Edinburgh. http:// www.rcsed.ac.uk/site/469/default.aspx. Visited 30 May 2006.

4. Webster's Online Dictionary. http://www.websters-online-dictionary.org/. Visited 30 May 2006.

5. What is informatics? Website of the Indiana University of Informatics. http://www.informatics. indiana.edu/overview/what is informatics.asp. Visited 30 May 2006

6. NHS careers in health informatics website. http://www.nhscareers.nhs.uk/details/Default. aspx? ld =767. Visited 30 May 2006.

7. NHS Connecting for Health website. www.connectingforhealth nhs.uk. Visited 30 May 2006.

8. Kurland L T, Molgaard C A. The patient record in epidemiology. Sci Am 1981; 245: 65-63.

9. Ledley R S, Lusted L B. Reasoning foundations of medical diagnosis. Science 1959; 130: 9-21.

10. Lusted L B. Medical progress - medical electronics. N Engl J Med 1955; 252: 580-585.

11. Collen M F, Davis L S, VanBrunt E E. The computer record in medical health screening. Methods Inf Med 1971; 10: 138-142.

12. NHS dentistry - options for change. London: Department of Health, 2002.

13. An Information technology strategy for NHS dentistry in the 21st Century. London: Department of Health, 2002.

14. Snow M G, Fallat R J, Tyler W R, Hsu S P. Pulmonary consult: concept to application of an expert system. J Clin Eng 1988; 13: 201-205.

15. Edwards G, Compton P, Malor R, Srinivasan A,
Lazarus L. PEIRS: a pathologist maintained expert system for the interpretation of chemical pathology reports. Pathology 1993; 25: 27-34.

16. Bizovi K E, Beckley B E, McDade M C et al. The effect of computer-assisted prescription writing on emergency department prescription errors. Acad Emerg Med 2002; 9: 1168-1175.

17. Bates D W, Leape L L, Cullen D J et al. Effect of computerized physician order entry and a team intervention on prevention of serious medication errors. JAMA 1998; 280: 1311-1316.

18. Kaplan B. Evaluating informatics applications: review of the clinical decision support systems literature. Int J Med Inf 2001; 64: 15-37.

19. Hunt D L, Haynes R B, Hanna S E, Smith K. Effects of computer-based clinical decision support systems on physician performance and patient outcomes: a systematic review. JAMA 1998; 280: 1339-1346.

20. National Electronic Decision Support Taskforce. Electronic decision support for Australia's health sector: report to health ministers by the National Electronic Decision Support Taskforce. Australia: Department of Health and Ageing, 2003.

21. Sim I, Gorman P, Greenes R A et al. Clinical decision support systems for the practice of evidencebased medicine. J Am Med Inform Assoc 2001; 8: $527-534$.

22. Swanson D R, Smalheiser N R. An interactive system for finding complementary literatures: a stimulus to scientific discovery. Artif Intell 1997; 91: 183-203.

23. Swanson D R. Fish oil, Raynaud's syndrome, and undiscovered public knowledge. Perspect Biol Med 1986; 30: 7-18.

24. Swanson D R. Migraine and magnesium: eleven neglected connections. Perspect Biol Med 1988; 31: 526-557. 